\title{
A Nonparametric Bayesian Approach to Estimating Malaria Prophylactic Effect After Two Treatments
}

\author{
Cletus Kwa Kum ${ }^{1,2, *}$, Daniel Thorburn ${ }^{1}$, Gebrenegus Ghilagaber ${ }^{1}$, Pedro Gil $^{3}$ and \\ Anders Björkman ${ }^{4}$
}

${ }^{1}$ Department of Statistics, Stockholm University, Sweden

${ }^{2}$ Mathematics/Computer Science Department, Faculty of Science, P.O. Box 67 Dschang, University of Dschang, Cameroon

${ }^{3}$ Drug Resistance Unit, Division of Pharmacogenetics, Department of Physiology and Pharmacology, Karolinska Institute, Stockholm, Sweden

${ }^{4}$ Division of Infectious Diseases, Karolinska University Hospital, Stockholm, Sweden

\begin{abstract}
Two treatment regimens for malaria are compared in their abilities to cure and combat reinfection. Bayesian analysis techniques are used to compare two typical treatment therapies for uncomplicated malaria in children under five years, not only in their power to resist recrudescence, but also how long they can postpone recrudescence or reinfection in case of failure. We present a new way of analysing this type of data using Markov Chain Monte Carlo techniques. This is done using data from clinical trials at two different centres. The results which give the full posterior distributions show that artemisinin-based combination therapy is more efficacious than sulfadoxine-pyrimethamine. It both reduced the risk of recrudescence and delayed the time until recrudescence.
\end{abstract}

Keywords: Bayesian clinical trial, conditional survival posterior, drug resistance, efficacy, recurrence time, uncomplicated malaria, sulfadoxine-pyrimethamine.

\section{INTRODUCTION}

In practice, the choice of a model to represent a natural phenomenon is not made solely on mathematical grounds but by knowing something about biology, medicine and other disciplines. Survival models are usually triggered by observations from clinical trials or data resulting from other forms of experiments. Clinical trials often lead to time-to-event (survival) data leading to time-to-event analysis known as survival analysis.

Bayesian methods have become quite popular in modelling survival data. The attractiveness of this technique is the easy interpretation of results and drawing of conclusions. Information from previous studies can easily be incorporated through an informative prior distribution. In the absence of tangible prior information, Bayesian techniques are applied using vague priors that often yield results similar to classical maximum likelihood methods. The advantages of Bayesian techniques in clinical trials and in epidemiology are discussed in [1-4].

Malaria is a vector-borne killer disease that is taking the lives of many who live in the infested zones.

\footnotetext{
*Address correspondence to this author at the Department of Statistics, Stockholm University, SE-106 91 Stockholm, Sweden; Tel: +46 81620 00; Fax: +468167511; E-mail: cletus.kum.kwa@stat.su.se, kumkwah@yahoo.co.in
}

Treatment failure due to parasite resistance is a major concern. Research is on the increase towards getting the best combination therapy that will cure and reduce resistance. Antimalarial drug efficacy clinical trials are galvanized. One of such trials was conducted in Tanzania in 2004 to compare the efficacy of two malaria treatments. These treatments were artesunate plus sulfadoxine-pyrimethamine (ASP) and sulfadoxine-pyrimethamine (SP) as an alternative. The ASP is an example of artemisinin-based combination therapy (ACT) and their use is encouraged by the World Health Organization. The concept of ACTs is well discussed in the works of [5-9]

We will exemplify our method using the data from the Tanzania study, where children suffering from malaria were randomly assigned to one of the two treatments which both were supposed to remove all parasites in the blood. All children were then monitored for 84 days. During follow-up dates and at emergency times in-between follow-ups [8], some patients were found to be carriers of malaria parasites. Other aspects of this study have been analysed in [10]. These parasites could have been due to recrudescence or reinfection. In a reinfection, the human host picks up an infectious agent again and experiences a new infection. Meanwhile a recrudescence is the recurrence of an infection which has been treated but only temporarily been suppressed to subpatent level. 
Distinguishing between a new infection and a recrudescence is relatively easier to be made in antimalarial trials in areas of low intensity than areas of high intensity [11]. There is always a probability, especially in malaria endemic areas, of misclassifying a reinfection as a recrudescence. Moreover, inhabitants of these areas are host to many different parasite genotypes.

Many studies have concluded that ACTs are better treatment therapies in terms of efficacy, but in exactly how long the therapies delay reinfection or recrudescence in case of failure has not been given the desired attention in modelling. Some related works in this direction for example [12] and [13], only based their studies on measuring parasitaemia over predefined periods to measure the duration for parasite clearance in the host's system.

In this paper we propose a method to determine which of the two treatments is more efficacious, and present a new methodology that can be used to estimate how long a treatment can postpone the recurrence of the disease in case of failure. We do not assume any distribution in determining the difference in mean survival times between the two treatments. The prophylactic effect after treatment terminology is used here, to capture results from the cure rate model and delay time to a first recurrence of disease model. We consider the first recurrence of malaria during follow-up periods as a heuristic justification of treatment failure in our analysis. Throughout this paper, to avoid the ambiguity in differentiating between malaria due to reinfection and recrudescence, all first appearance of malaria within the study period are known simply as a first recurrence of malaria. The mean survival time for patients receiving each of the two regimens before a first recurrence of malaria is considered as the mean survival time for each of these two therapies.

In the delay time model before a first recurrence of malaria, we only look at first recurrences of the disease before the last day of the study. After that day all recurrences are due to reinfections (new inoculations). All differences in recrudescence rates will thus be found as differences between the recurrence rates before day 84 . This supposition is important in order that our estimated posterior mean (difference) in survival times should not be biased as a consequence of the survival plots terminating prematurely due to censoring. A discussion on this can be found in [14, 15]. By survival mean time, we mean the truncated survival mean time defined by the period of the clinical trial and this is given by the area under the survival plot. Some authors, $[16,17]$ propose the method of extrapolation, but this procedure produces less accurate estimates. Moreover, this allusion was made when determining the mean survival time involving one function, and not the difference between two survival functions as in our case.

In our data, the participants were followed for 84 days after treatment. Our analyses do not focus on only the 84 days period but also on the first 42 days. Gbotosho [18] defined recrudescence as the reappearance of parasitaemia before day 42 and confirmed by polymerase chain reaction (PCR). After some time, reinfection cases start appearing but it is not clear when recrudescence no longer can appear. As a result, we repeat our analyses assuming Day 42 as the period of the trials in order to see the difference.

In our approach, it is quite possible to estimate the distribution of the time until reinfection for both treatments and then look at the difference. But in order to be able to decide whether the distributions differ, we focus on the change in delay time. We use the truncated survival analysis procedure to obtain posterior distributions and posterior mean estimates for the delay time to a first recurrence of malaria at the chosen periods. As a bonus, it turns out that these posterior distributions look fairly normal even though the expected times till first recurrence does not.

Applying models to malaria data, we computed posterior survival probabilities of the success of each treatment therapy within 42 days and 84 days. We obtained delay time estimates for ASP and SP together with their full posterior densities for treatment efficacies. Also we obtained estimates and posterior densities for overall efficacy of treatment, infection rate per follow-up and delay time to a first recurrence of the disease. The results confirmed the results of previous studies that the efficacy of artemisinin-based combination therapy is better than non-combination therapies. With our new methodology, we obtained posterior estimates and full posterior densities for delay times before a malaria recurrence for the combination therapy.

The rest of the paper is organized as follows. We formulate the statistical models in Section 2. In Section 3 , we apply our methodology to data sets. Section 4 contains our results and the paper ends with a discussion in Section 5. 


\section{THE STATISTICAL MODELS}

\subsection{Cure Rate Model}

Let $n_{i}$ be the number at the start receiving treatment $i$ and let $X_{i}$ be the random variable describing the number of cured patients (without malaria before time $t_{\max }$, the end of the follow-up). Then our model is $X_{i} \sim \operatorname{Bin}\left(n_{i}, p_{i}\right)$, where $i=\mathrm{ASP}$ or SP and $p_{i}$ is the probability to be cured by treatment $i$.

Assuming that the prior for $p_{i}$ is the conjugate distribution $\operatorname{Beta}(\alpha, \beta)$, the posterior distribution for $p_{i}$ is

$\operatorname{Beta}\left(\alpha+X_{i}, \beta+n_{i}-X_{i}\right)$ for $i=$ ASP or SP .

In the application we will assume that there is no prior information on the probabilities and thus use two independent Jeffreys priors with $\alpha=\beta=1 / 2$. With independent priors, the posteriors will also be independent. It is quite common to use a Jeffreys prior in this situation.

Since we do not want to assume any knowledge before the study, a prior distribution with minimal information is chosen. The uniform prior $\operatorname{Beta}(\alpha=0, \beta=0)$ and the Jeffreys prior are good examples. Discussions on prior distributions can be found in [19-23].

In the following subsections, we describe and develop a model that can be used in determining the difference in delay time between two treatments, before a first recurrence of a disease in a study. The disease we consider is malaria where the first recurrence may be due to a recrudescence or a reinfection.

\subsection{Recurrence Rate Model at Each Follow-Up}

Suppose that rescreening to determine the presence or absence of the disease is done at fixed time points, $t_{0}=0, t_{1}, \ldots, t_{k}=t_{\max }$. We make an important assumption that these time points are the same for the two treatments.

Let $R_{j, i}$ and $Y_{j, i}$ denote the number of children who had been free from malaria up to time point $t_{j-1}$ and those who get malaria between time points $t_{j-1}$ and $t_{j}$ ( $i=$ ASP or SP), respectively. In particular we have that $R_{j, i}+Y_{j, i}=R_{j-1}, i$. Then for each of these intervals, the children $Y_{j, i}$ witnessing the event of interest can be modelled as $\operatorname{Bin}\left(R_{j, i}, \theta_{j i}\right)$ where $\theta_{j i}$ stands for the conditional probability of becoming sick in this interval for those who have not had any recurrence at or before $t_{j-1}$. Immediately a child witnesses a first recurrence of malaria at any time point, she is censored and no longer considered to be at risk of recurrence for the rest of the time.

In the same way as in equation (1), the posterior distribution of $\theta_{j i}$ is

$\operatorname{Beta}\left(\alpha_{j i}+Y_{j, i}, \beta_{j i}+R_{j-1, i}-Y_{j, i}\right)$,

if the prior is Beta distribution with hyperparameters $\alpha_{i i}$ and $\beta_{i i}$. We will also assume that all binomial distributions and priors are independent for different intervals and treatments. Here, we equally use the Jeffreys prior, $\alpha=\beta=1 / 2$ for all intervals and all treatments.

The next step towards our goal of obtaining a posterior distribution for the difference in delay time for one of the treatments, is to get the posterior survival estimates at follow-ups.

\subsection{Survival Rate Model at Each Follow-Up}

The posterior distribution of $\theta_{j i}$ is given by model (2). Thus we have the posterior for the survival functions at all follow-up time instances for each treatment given by

$S\left(t_{j}\right)=\prod_{k=1}^{j}\left(1-\theta_{k i}\right)$

To obtain the full distribution, we assume that it is a piecewise linear function which is linear between the follow-up times $t_{0}=0, t_{1}, \ldots, t_{k}=t_{\max }$, where there are no observations to aid us. Thus we assume that those who get a recurrence between $t_{j-1}$ and $t_{j}$ at the average get a recurrence at the time midpoint $\left(t_{j-1}+t_{j}\right) / 2$.

We could also have assumed a constant hazard rate which would correspond to a slightly shorter mean time, $t_{j-1}-\left[\left(t_{j}-t_{j-1}\right)\left(\frac{1-\theta_{j i}}{\theta_{j i}}+\frac{1}{\operatorname{In}\left(1-\theta_{j i}\right)}\right)\right]$ but since our values are fairly small the difference is negligible.

\subsection{Difference in Delay Time to First Recurrence}

Let the random variable $T$ represents the survival time until first recurrence of the disease. Assuming that $T$ is continuous with density $f(s)$, then the probability of surviving the event of interest till time $t$ is given by the survival function 
$S(t)=1-F(t)=\int_{t}^{\infty} f(s) d s$.

The mean survival time for $T$ with probability density function $f(s)$ is defined as

$E(T)=\int_{0}^{\infty} t f(s) d s=\int_{0}^{\infty} S(s) d s$.

This mean time is given by the area under the survival function. If the distribution is bounded, the survival function tends to zero at that time. If this is not the case, other methods such as the extrapolation technique can be used but will lead to less accurate measures, [17].

Complete survival curves are not usually observed during studies due to time constraints and costs. The methodology we develop imposes the condition that the event of a first recurrence must be observed before time $t_{\max }$. However, the formula we present still takes into account both observed and unobserved parts of the survival curve. Thus for a given time $t_{k}=t_{\max }<\infty$, the complete area under the curve (for each treatment $i$ ), which corresponds to the mean time is given by

$$
\begin{aligned}
E(T)= & \sum_{j}\left[\left(t_{j}-t_{j-1}\right) S\left(t_{j}\right)+\left(S\left(t_{j-1}\right)-S\left(t_{j}\right)\right)\left(t_{j}-t_{j-1}\right) / 2\right] \\
& +E\left(\max \left(T-T_{\max }, 0\right)\right) \\
= & \sum_{j}\left[\left(t_{j}-t_{j-1}\right)\left(S\left(t_{j}\right)+S\left(t_{j-1}\right)\right) / 2\right]+E\left(\max \left(T-T_{\max }, 0\right)\right)
\end{aligned}
$$

The last term in the sum in equation (4) is the healthy time in the period for those who get sick within the same period. The last term outside the sum corresponds to the excess time of those who are healthy at the end of the follow-up.

For $t_{j}<t_{\max }$, the conditional survival function of those that have a first recurrence (recrudescence or reinfection) is given by

$S *\left(t_{j} \mid T<t_{\max }\right)=\frac{\prod_{k=1}^{j}\left(1-\theta_{k i}\right)}{1-S\left(t_{\max }\right)}$.

The expected delay time conditional on a recurrence before time $t_{\max }$ is thus given by,

$$
\begin{aligned}
& E\left(T \mid T<t_{\max }\right)=\frac{1}{2} \sum_{j}\left(t_{j}-t_{j-1}\right)\left[S *\left(t_{j} \mid T<t_{\max }\right)\right. \\
& \left.+S *\left(t_{j-1} \mid T<t_{\max }\right)\right]
\end{aligned}
$$

Now, suppose that $T_{1}$ and $T_{2}$ represent survival times until the reccurrence of the event of interest in treatment groups $1(=\mathrm{ASP})$ and $2(=\mathrm{SP})$, respectively.
We want to know how much longer $T_{1}$ is expected compared with $T_{2}$. The expected difference between the two survival times is

$$
E\left[T_{1}-T_{2}\right]=E\left[\int_{0}^{\infty} S_{1}\left(t_{1}\right) d t_{1}-\int_{0}^{\infty} S_{2}\left(t_{2}\right) d t_{2}\right]
$$

This equation defines the area between the two survival curves $S_{1}$ and $S_{2}$, if $T_{1}$ is stochastically larger than $T_{2}$.

Given that $T_{1}<T_{\max }$ and $T_{2}<T_{\max }$ the observed expected difference can then be estimated by

$$
E\left[T_{1}-T_{2}\right]=E\left[T_{1} \mid T_{1}<t_{\max }\right]-E\left[T_{2} \mid T_{2}<t_{\max }\right],
$$

where $E\left[T_{i} \mid T_{i}<t_{\max }\right]$ is as defined in equation (6). We note that the prior for $\theta_{j i}$ induces corresponding priors for all other measures of interest. Hence, the posterior expected delay time for the two treatments is given by

$\frac{1}{2} \sum_{j}\left(t_{j}-t_{j-1}\right)\left[\left(S_{1}^{*}\left(t_{j} \mid \tilde{T}\right)+S_{1}^{*}\left(t_{j-1} \mid \tilde{T}\right)\right)-\left(S_{2}^{*}\left(t_{j} \mid \tilde{T}\right)+S_{2}^{*}\left(t_{j-1} \mid \tilde{T}\right)\right)\right]$

This can further be simplified to

$$
E\left[T_{1}-T_{2} \mid \tilde{T}\right]=\frac{1}{2} \sum_{j}\left[\left(t_{j}+t_{j-1}\right)\left(S_{1}^{*}\left(t_{j} \mid \tilde{T}\right)-S_{2}^{*}\left(t_{j} \mid \tilde{T}\right)\right)\right],
$$

where $\tilde{T}=T<T_{\max }$, We note that $E\left(\max \left(T-T_{\max }, 0\right)\right)=0$ since $t_{k}=t_{\max }$ must not be an event time in the model assumption.

\subsection{Monte Carlo Implementation}

We use the MCMC Gibbs sampler in the Bayesian setup. For the efficacy posterior estimates and densities, we draw random samples from their posterior distributions defined in Section 2.1.

The model given by expression (7), involves a complex posterior distribution we call $G$ that links $(2 \times(k-1))$ Beta distributions corresponding to the two treatments and $k$ follow-up times. The procedure is as follows:

Sample $\theta_{j i}^{(1)}$ from $\operatorname{Beta}\left(Y_{j, i}+\alpha, R_{j, i}-Y_{j, i}+\beta\right)$, then compute $S_{j i}^{*(1)}$ and $G^{(1)}, \ldots$, Sample $\theta_{j i}^{(N)}$ from $\operatorname{Beta}\left(Y_{j, i}+\alpha, R_{j, i}-Y_{j, i}+\beta\right)$ compute $S_{j i}^{*(N)}$ and $G^{(N)}$. The resulting sequence $\left\{G^{(1)}, G^{(2)}, \ldots, G^{(N)}\right\}$ constitutes $N$ independent samples from $G$. A histogram is plotted for these simulations to obtain the posterior distribution for $G=E\left[T_{1}-T_{2} \mid().\right]$. 
Table 1: Number with First Recurrence of Malaria and those at Risk (in parentheses)

\begin{tabular}{|c|c|c|c|c|c|c|c|}
\hline \multirow{2}{*}{ Location } & Drug & $\mathbf{( 0 - 7 )}$ & $\mathbf{( 7 - 2 1 )}$ & $\mathbf{( 2 1 - \mathbf { 2 8 } )}$ & $\mathbf{( 2 8 - 4 2 )}$ & $\mathbf{( 4 2 - 5 6 )}$ & $\mathbf{( 5 6 - \mathbf { 8 4 } )}$ \\
\hline \hline \multirow{2}{*}{ KONDE } & $\mathrm{ASP}$ & $3(90)$ & $8(87)$ & $21(79)$ & $16(58)$ & $7(42)$ & $3(35)$ \\
\cline { 2 - 7 } & $\mathrm{SP}$ & $7(86)$ & $17(79)$ & $10(62)$ & $9(52)$ & $10(43)$ & $4(33)$ \\
\hline \multirow{2}{*}{ UZINI } & $\mathrm{ASP}$ & $4(94)$ & $13(90)$ & $9(77)$ & $8(68)$ & $9(60)$ & $1(51)$ \\
\cline { 2 - 7 } & $\mathrm{SP}$ & $15(110)$ & $14(95)$ & $13(81)$ & $6(68)$ & $2(62)$ & $3(60)$ \\
\hline
\end{tabular}

\section{APPLICATION TO MALARIA}

\subsection{Description of Data}

Our data came from a clinical trial conducted in 2004 in Tanzania to compare the efficacy of artesunate plus sulfadoxine-pyrimethamine and sulfadoxinepyrimethamine alone in treating children under five years that were infected with Plasmodium falciparum malaria parasites. The study was done in two villages, Konde and Uzini, with a total of 178 and 206 patients, respectively. Children enrolled in these trials were randomly assigned to receive either of these therapies. The children had to meet certain entry requirements; the most important was having malaria with no other complications, and being willing to partake in the study. The children were tested again for malaria at days 7 , $21,28,42,56$ and 84 after treatment.

Table 1 presents our data on the number of patients having a first recurrence of malaria at the specified dates or intervals. There were two missing observations in each of the two data sets. We could not distinguish between malaria due to recrudescence or reinfection (new infection). Our focus is on the number of patients who were diagnosed as sick again. There are probably a few new infections but almost all cases are most likely due to recrudescence. We consider only the first recurrence of malaria.

We applied the models described in Section 2, to the given data. We considered two study periods; a period that starts from Day 0 to Day 42 and another period that starts from Day 0 to Day 84 . The choice of these periods was motivated by the fact that some clinical trials do last for 42 days, while others last for 84 days. The explanation given by some medics is that within 42 days, the effect of the most long lasting drugs (long elimination half-life) must have become negligible so that most suppressed recrudescences should have had the opportunity to reappear. Meanwhile the 84 days period is to explore if or ensure that the foregoing assumption was correct. The total rate of recurrences per week was $28 / 207 \times 2=7 \%$ between days 42 and 56 , and $11 / 179 \times 4=2 \%$ in the last period. This indicates that most of the cases in-between days 42 and 56 must have been recrudescence, since the rate of cases due to reinfection should be rather stable between the periods. We take $t_{\max }=42$ days, since other authors have used that, and 84 days, since there seems to be many cases of recrudescence also after day 42 . For consistency, we maintain the terminology of a first recurrence over a first recrudescence or first reinfection. The next subsections explain the application procedure.

\subsection{Treatment Efficacy}

Some patients never experienced a recurrence of malaria throughout the two study periods. This is the category we considered as having been successfully treated by the therapies they received. The simple model described in Section 2.1 is applicable here.

\subsection{First Recurrence and Survival Rates at Follow-Ups}

As shown in Table 1, there were six designated intervals to monitor malaria symptomatic patients during the whole period of trials. A quick observation here is that the interval lengths between follow-ups are not of equal length. Notwithstanding, this is usually the case in practice. Moreover, intervals between followups and the duration of trials depend on the nature of the disease studied [24]: 112, and should last as long as the duration of the treatment requires. The methodology presented here takes care of this by assuming that such equidistant points lie on lines joining two adjacent known recurrence rate points.

Knowing the recurrence rates at each time point, provide us with the necessary information for obtaining the conditional survival probability estimates for surviving upto the given time points. The delay time model is conditioned on no first recurrence beyond time $t_{\max }$. By implication, this means $S\left(t_{j} \mid t_{j} \geq t_{\max }\right)=0$ as shown in equation (5).

\subsection{Delay Time Before First Recurrence by Each Treatment}

The escape (survival) of patients from a first instance of malaria largely depends on the treatment regimen taken. There is a general consensus based on 
Table 2: Posterior Estimates for Overall Treatment Efficacy

\begin{tabular}{|c|c|c|c|c|c|c|c|}
\hline Location & Period & Parameter & Estimate & SE & $2.5 \%$ & $50 \%$ & $97.5 \%$ \\
\hline \multirow{2}{*}{ KONDE } & \multirow{2}{*}{$(0-42]$} & $P_{A S P}$ & 0.51 & 0.05 & 0.41 & 0.51 & 0.61 \\
\hline & & $P_{S P}$ & 0.50 & 0.05 & 0.40 & 0.50 & 0.60 \\
\hline \multirow{2}{*}{ UZINI } & \multirow{2}{*}{$(0-42]$} & $P_{A S P}$ & 0.67 & 0.05 & 0.57 & 0.67 & 0.76 \\
\hline & & $P_{S P}$ & 0.56 & 0.05 & 0.47 & 0.56 & 0.65 \\
\hline \multirow{2}{*}{ KONDE } & \multirow{2}{*}{$(0-84]$} & $P_{A S P}$ & 0.38 & 0.05 & 0.28 & 0.38 & 0.48 \\
\hline & & $P_{S P}$ & 0.34 & 0.05 & 0.24 & 0.34 & 0.44 \\
\hline \multirow{2}{*}{ UZINI } & \multirow{2}{*}{$(0-84]$} & $\mathrm{P}_{\mathrm{ASP}}$ & 0.59 & 0.05 & 0.49 & 0.60 & 0.69 \\
\hline & & $\mathrm{P}_{\mathrm{SP}}$ & 0.52 & 0.05 & 0.43 & 0.52 & 0.61 \\
\hline
\end{tabular}

earlier studies that ACTs are better than alternative therapies used for the treatment of uncomplicated malaria. It will be interesting to know for how long ASP can postpone a recurrence when it cannot effectively treat patients. Model given by equation (7) is suited for this purpose.

\subsection{Estimation Procedure}

The MCMC procedure described earlier were applied, using $N=1000000$ independent samples. The required posterior estimates, plots and densities were obtained.

\section{RESULTS OF SIMULATIONS}

The results from simulations are presented in Tables 2, $\mathbf{3}$ and $\mathbf{4}$. Figures $\mathbf{1}$ to $\mathbf{8}$, present posterior probability distributions for the cure rates, survival plots and the expected difference in delay times. In each case, we have two periods: $(0-42)$ days and $(0-84)$ days.

\subsection{Efficacy Estimates and Posterior Distributions}

Table 2 shows the posterior estimates for the performance (efficacy) of each treatment within 42 and 84 days, respectively. The estimates are for the parameters $P_{A S P}$ and $P_{S P}$ which represent the posterior probabilities of successful cure with the treatments ASP and SP, respectively. We observe that in each treatment centre and for each trial period, the mean of effective cure with ASP is higher. Accompanying measures are the standard errors and the endpoints of the $95 \%$ credible interval. Table 3 lay out the posterior estimates for the probability of ASP performing better than SP $\left(P_{A S P}>P_{S P}\right)$ from our simulations, for each study location and period. The probability is above $70 \%$ except for the Konde 42 days duration.

Table 3: Posterior Estimates: $\mathrm{P}(\mathrm{ASP}>\mathrm{SP})$

\begin{tabular}{|c|c|c|}
\hline Period & KONDE & UZINI \\
\hline \hline$(0-42]$ & 0.55 & 0.94 \\
\hline$(0-84]$ & 0.71 & 0.87 \\
\hline
\end{tabular}

The corresponding full posterior distributions for treatment efficacy of the two treatments are given in Figures 1 and 2. The posteriors for ASP lie to the right of that of SP. This means there are higher chances of getting a cure using ASP than SP. However, the Konde 42 days duration shows a small shift in favour of ASP but the difference between the two therapies here is negligible.

\subsection{Posterior Survival Plots}

We now consider plots found in Figures 3 to 6 . Figures $\mathbf{3}$ and $\mathbf{5}$ present the observed posterior survival time plots before the event of a first recurrence at both Konde and Uzini. At the start of the trial, the treatments had equal survival probability 1 , of escaping the event of first recurrence of malaria. These plots show a steady decrease in survival probabilities through the length of each study period. We observe that more often, the plot for artesunate plus sulfadoxinepyrimethamine is above that of sulfadoxinepyrimethamine, which is indicative of their abilities to resist a recurrence of the disease.

On the other hand, Figures 4 and 6 show the truncated posterior plots with $S *\left(t_{\max }\right)=0$. These are the resulting plots obtained when we impose the condition that if a first recurrence is to occur, this must take place within a specified time before the end of 
KONDE:TREATMENT EFFICACY IN 42 DAYS

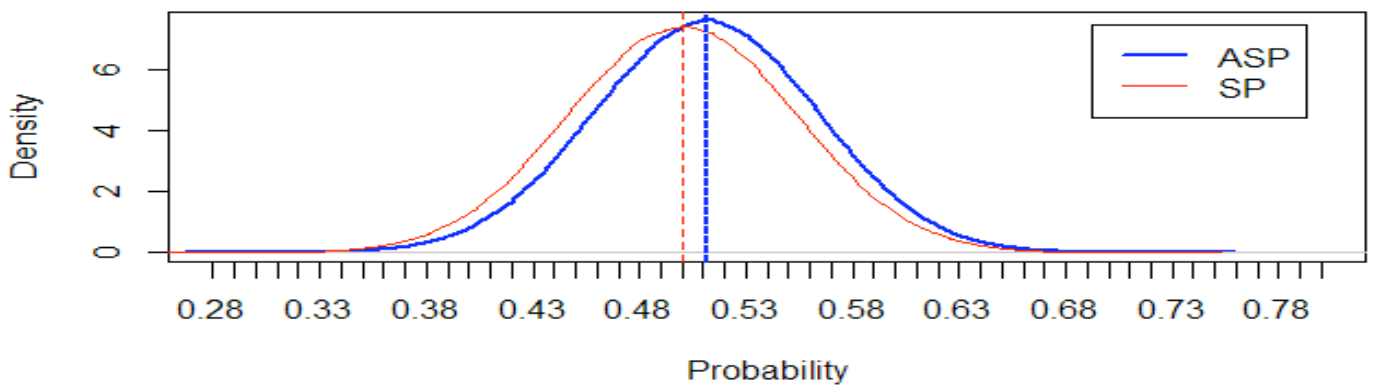

UZINI: TREATMENT EFFICACY IN 42 DAYS

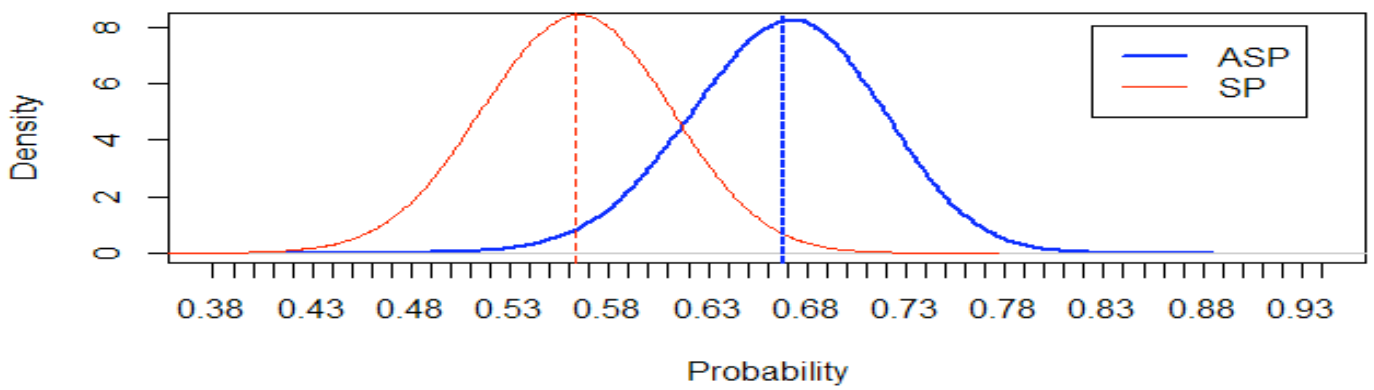

Figure 1: Treatment Efficacy after 42 days Posterior Distribution.

KONDE:TREATMENT EFFICACY IN 84 DAYS

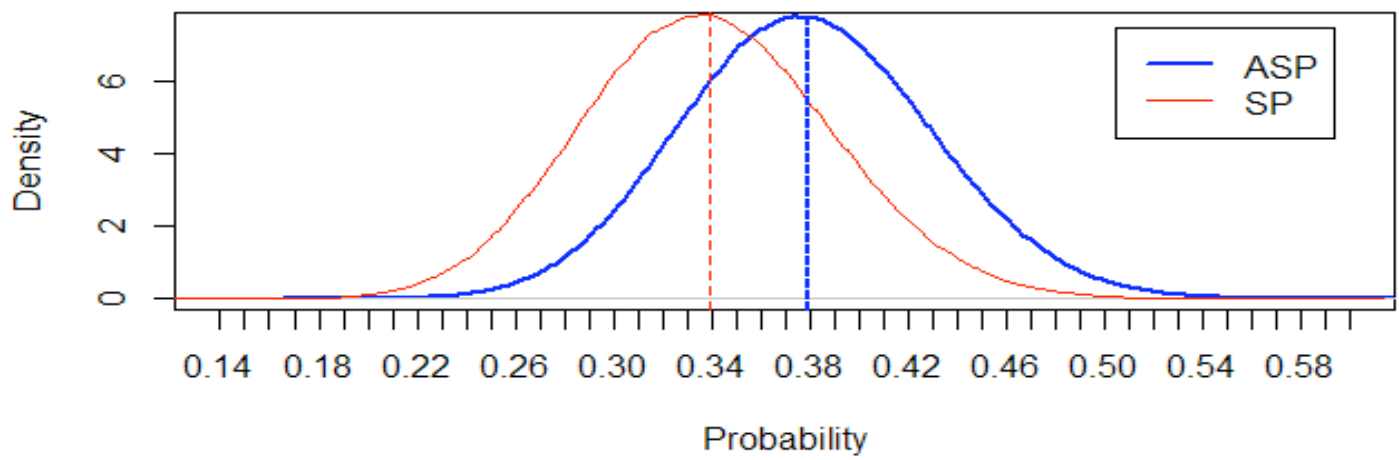

UZINI: TREATMENT EFFICACY IN 84 DAYS

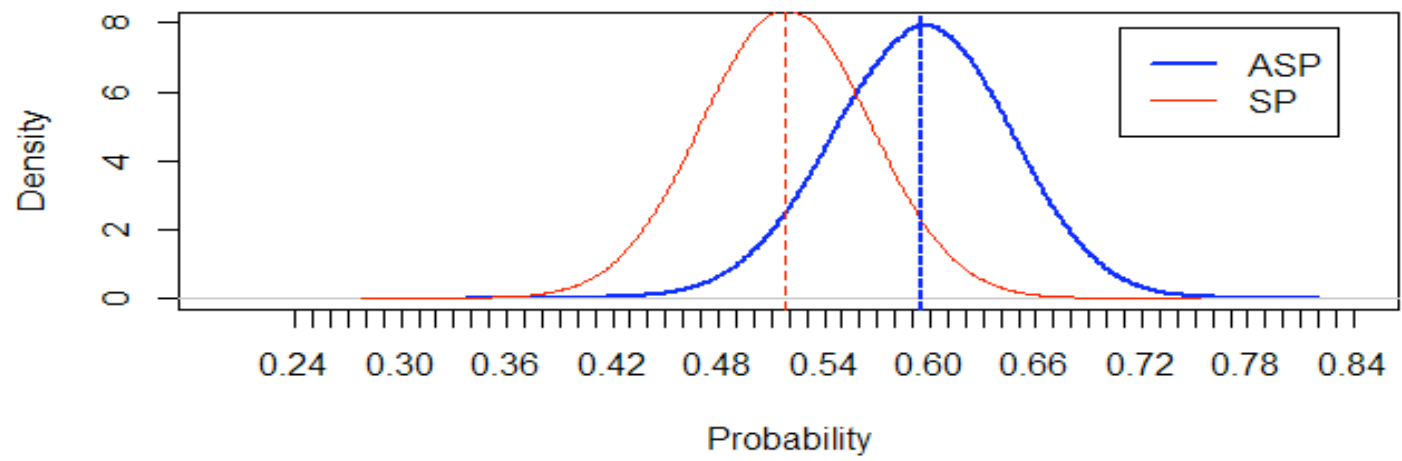

Figure 2: Treatment Efficacy after 84 days Posterior Distribution. 
KONDE: SURVIVAL PLOTS IN 42 DAYS

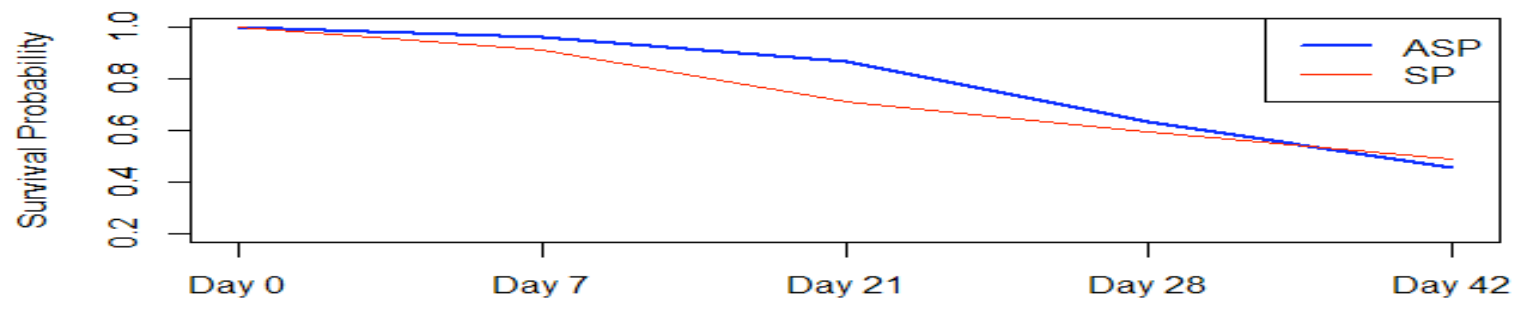

Follow-up time

UZINI: SURVIVAL PLOTS IN 42 DAYS

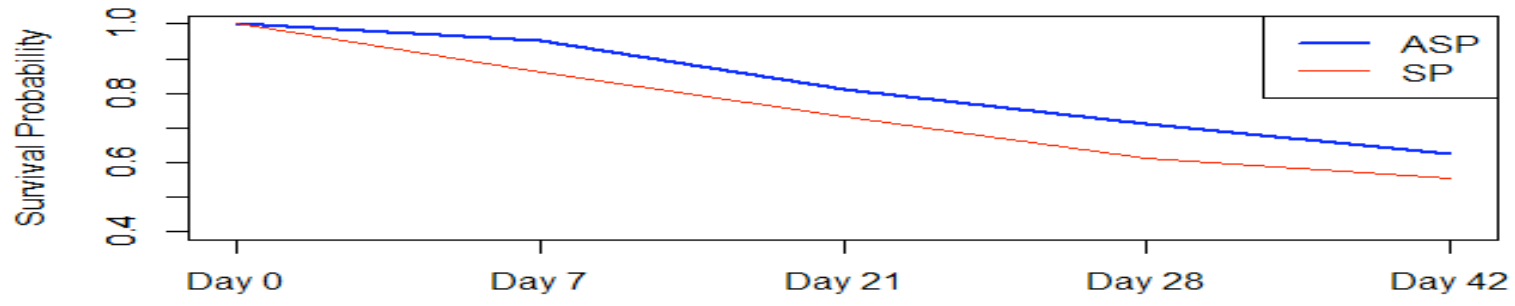

Follow-up time

Figure 3: Observed 42 days posterior survival plots.

KONDE: DAY 42 TRUNCATED SURVIVAL PLOTS

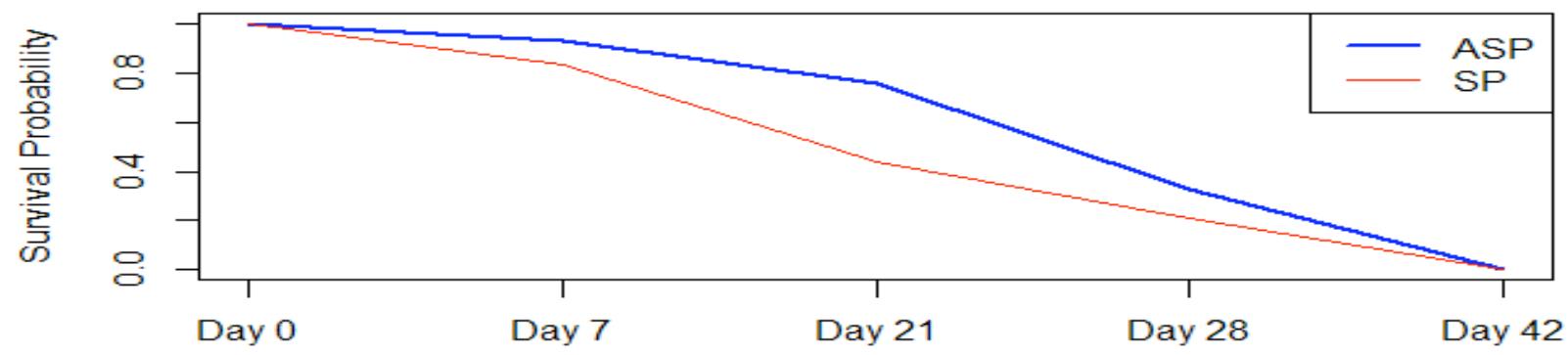

Follow-up time

UZINI: DAY 42 TRUNCATED SURVIVAL PLOTS

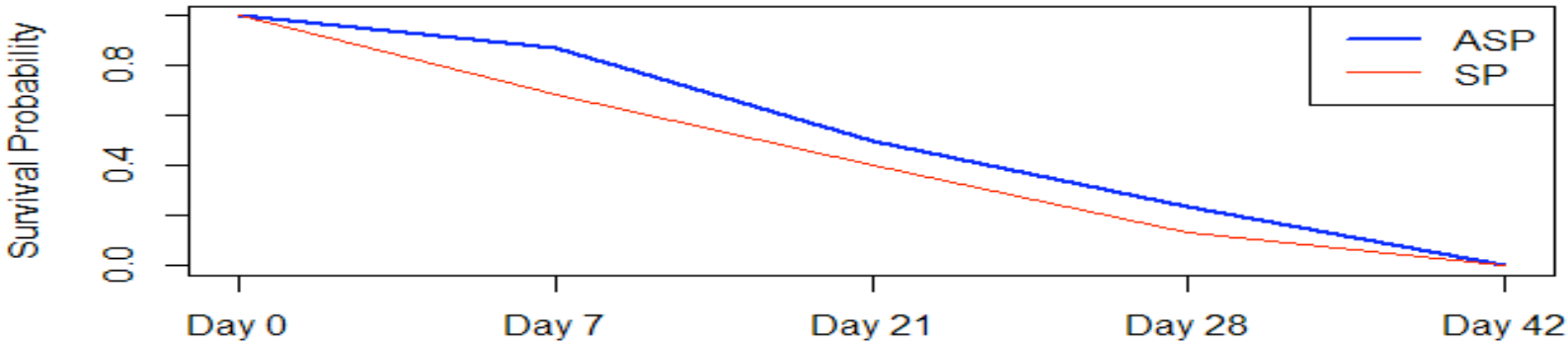

Follow-up time

Figure 4: Truncated 42 days posterior survival plots.

each trial period. In this paper we chose within 42 days and within 84 days. The truncated survival plots are important if we are to correctly estimate the posterior distribution of the difference in survival times. Moreover, it is usually difficult to keep track of events after the trials. 
KONDE: SURVIVAL PLOTS IN 84 DAYS
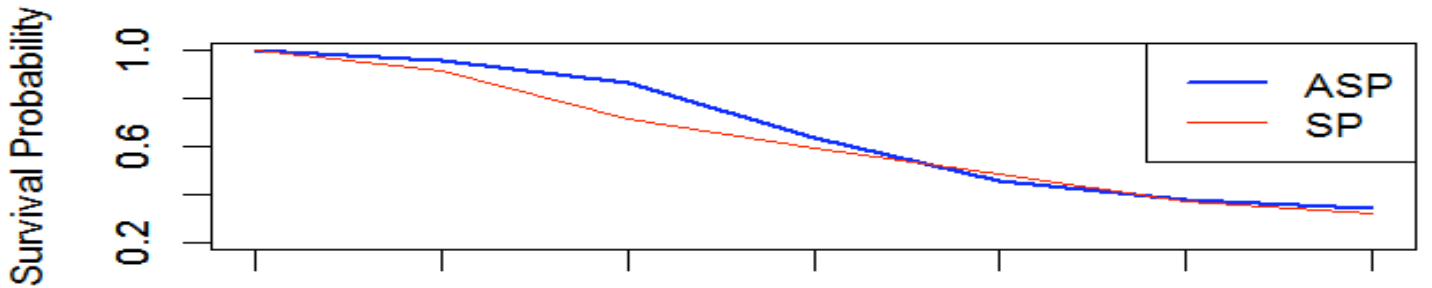

Day 0 Day 7 Day 21 Day 28 Day 42 Day 56 Day 84

Follow-up time

\section{UZINI: SURVIVAL PLOTS IN 84 DAYS}

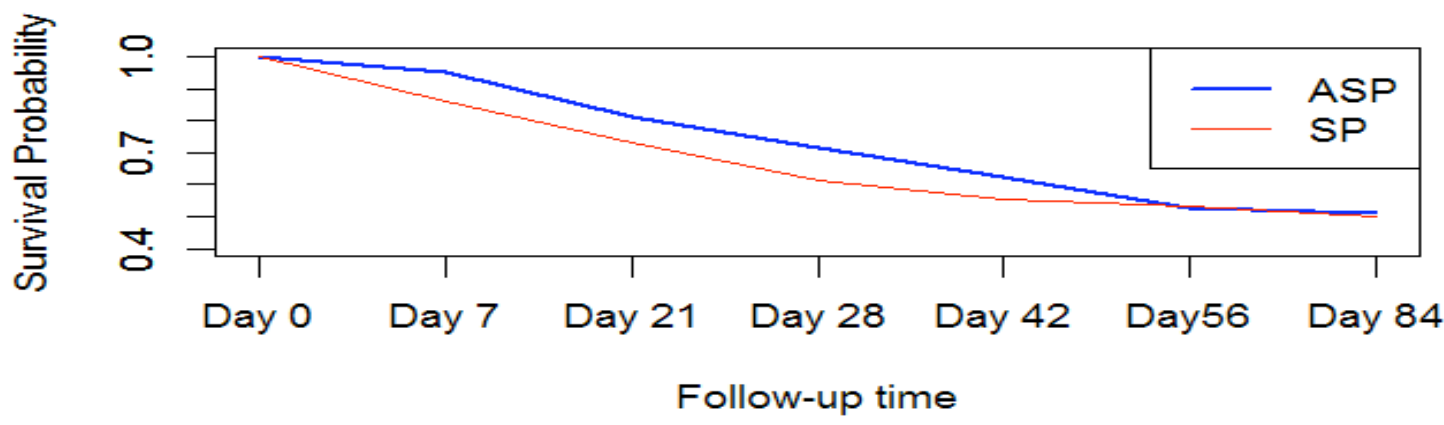

Figure 5: Observed 84 days posterior survival plots.

KONDE: DAY 84 TRUNCATED SURVIVAL PLOTS
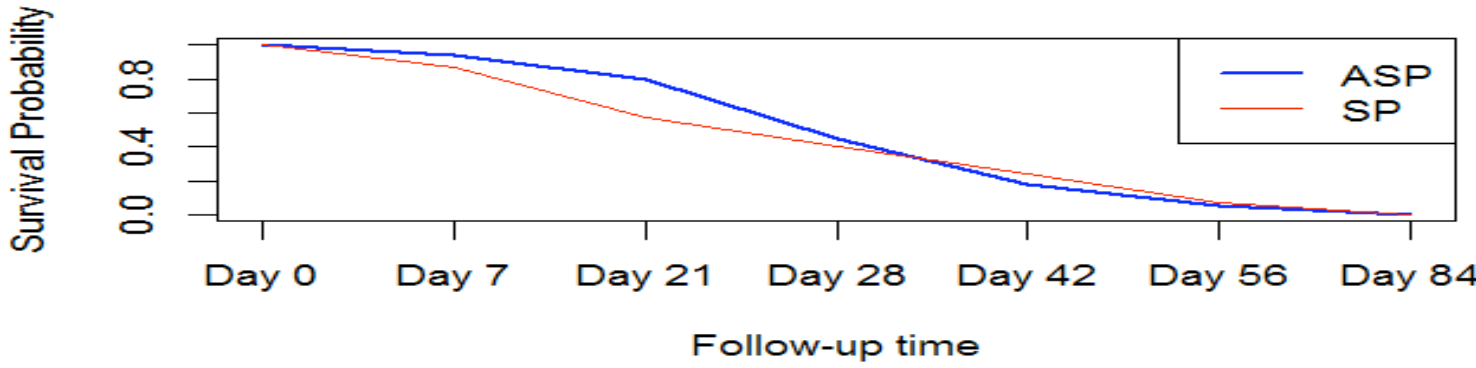

UZINI: DAY 84 TRUNCATED SURVIVAL PLOTS
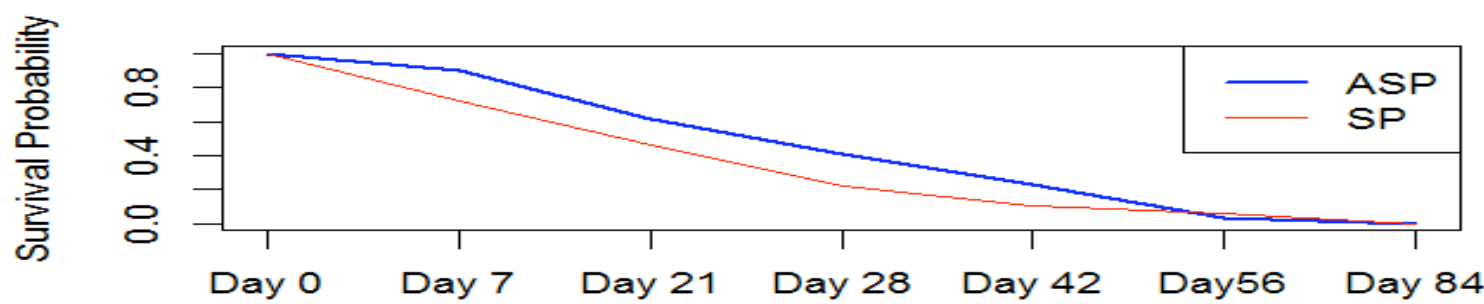

Follow-up time

Figure 6: Truncated 84 days posterior survival plots.

\subsection{Delay Time to First Recurrence of Malaria}

The area between the truncated survival curves is a measure of the expected difference in adjourn times before the event of a first recurrence for ASP. The estimates at the two study sites within 42 and 84 days are given in Table 4 . The mean estimates for the 42 
Table 4: Posterior Estimates for Mean Delay by ASP

\begin{tabular}{|c|c|c|c|c|c|c|c|}
\hline Location & Period & Parameter & Estimate & SE & $2.5 \%$ & $50 \%$ & $97.5 \%$ \\
\hline \multirow{2}{*}{ KONDE } & $0-42$ & $\mu_{\mathrm{k} 1}$ & 6.38 & 1.50 & 3.36 & 6.40 & 9.23 \\
\hline & $0-84$ & $\mu_{\mathrm{k} 2}$ & 2.98 & 2.73 & -2.43 & 3.00 & 8.28 \\
\hline \multirow{2}{*}{ UZINI } & $0-42$ & $\mu_{\mathrm{u} 1}$ & 6.11 & 1.46 & 3.18 & 6.14 & 8.90 \\
\hline & $0-84$ & $\mu_{\mathrm{u} 2}$ & 7.78 & 2.70 & 2.34 & 7.83 & 12.96 \\
\hline \multirow{2}{*}{ KONDE UZINI } & $0-42$ & $\mu_{\mathrm{ku}}$ & 6.24 & 1.05 & 4.20 & 6.24 & 8.29 \\
\hline & $0-84$ & $\mu_{\mathrm{ku}}$ & 5.41 & 1.92 & 1.64 & 5.41 & 9.17 \\
\hline
\end{tabular}

days running period are 6.38 in Konde and 6.11 in Uzini, respectively are quite closed. Meanwhile, there is a disparity in estimates for the 84 days trial period. The mean delay times were 2.98 in Konde and 7.28 in Uzini, respectively.

To have an overall picture of the delay times, we obtained the posterior distributions for the chosen two periods at both centres. The distributions are displayed in Figures $\mathbf{7}$ and 8. The posterior histograms obtained, are fairly Gaussian with great overlapping within the 42 days period at both centres. Though there is a horizontal shift when it comes to within 84 days period densities, the overlapping is still within 2 standard deviations.

We therefore assumed normality in delay time to the event of a first recurrence and used the Bayesian joint posterior for the mean, to have a single (combined) estimate for the mean delay time and its variance for the two centres. Thus $\hat{\mu}_{k u}=\frac{\left(\hat{\mu}_{k} \hat{\sigma}_{k}^{-2}+\hat{\mu}_{u} \hat{\sigma}_{u}^{-2}\right)}{\left(\hat{\sigma}_{k}^{-2}+\hat{\sigma}_{u}^{-2}\right)}$ where $\hat{\sigma}_{\hat{\mu} k u}^{-2}=\left(\hat{\sigma}_{k}^{-2}+\hat{\sigma}_{u}^{-2}\right)^{-1}$ are the combined mean and variance estimates for the two study sites. These mean estimates are 6.24 days and 5.41 days with variances 1.10 and 3.68 for trials lasting within 42 and 84 days, respectively. These results are on the last two rows in Table 4.

\section{DISCUSSION}

We proposed a new methodology that can be used in the estimation of how long one of two therapies can postpone or delay the first recurrence of a disease using a Bayesian approach through MCMC techniques. We demonstrated the performance of the methodology by applying it to data on efficacy of two malaria treatments in a clinical trial. Although we used the malaria data with two treatments, the methodology can be extended to other similar studies.
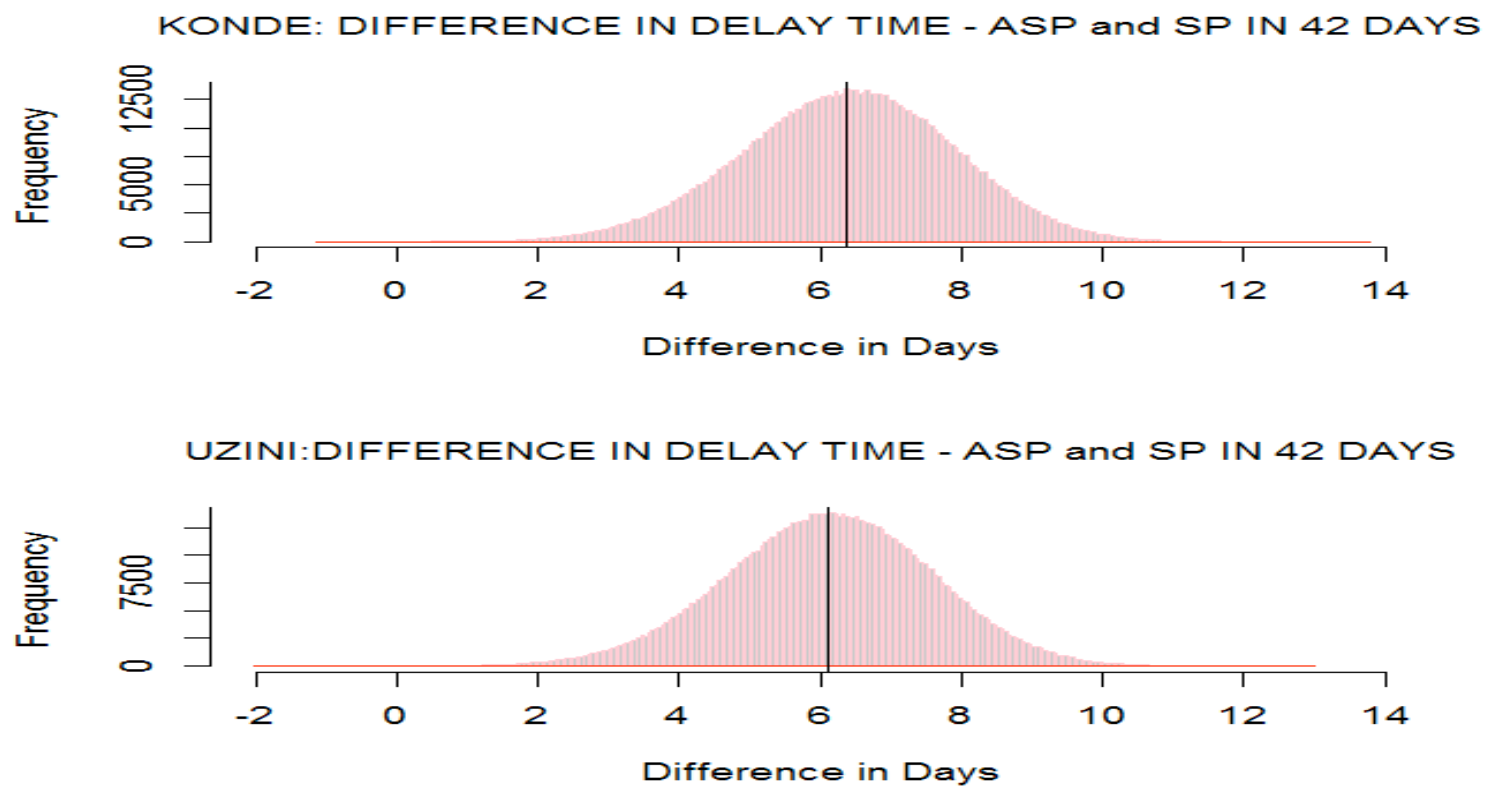

Figure 7: Treament Efficacy after 42 days Posterior Distribution. 
KONDE: DIFFERENCE IN DELAY TIME - ASP and SP IN 84 DAYS

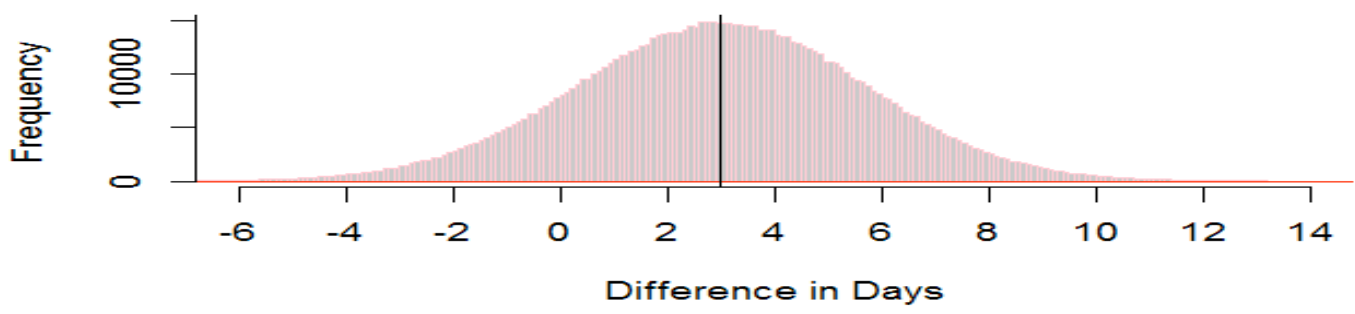

UZINI:DIFFERENCE IN DELAY TIME - ASP and SP IN 84 DAYS

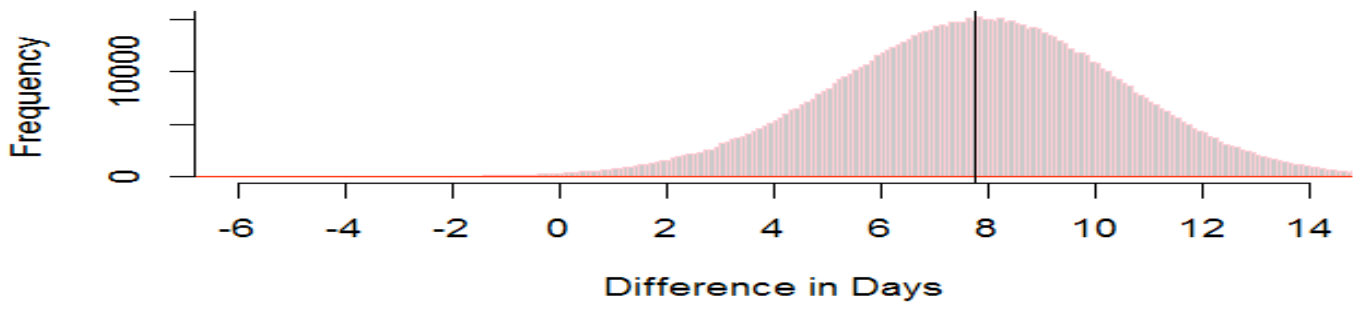

Figure 8: Treatment Efficacy after 84 days Posterior Distribution.

A significant profit of the Bayesian approach is that it provides a complete distribution for parameters of interest. We had parameters on overall cure rate or treatment efficacy, first recurrence and survival rates per follow-up and the expected difference in delay before a recurrence of malaria. We made a key assumption that all recrudescences take place before the last day of study. This assumption was necessary to circumvent the intractability of the event of interest beyond the study periods.

The results on the efficacy of treatment showed that artesunate plus sulfadoxine-pyrimethamine was a better treatment confirming the reasons behind the advocacy of artemisinin-based combination therapy for the treatment of malaria. However, we observed some discrepancies in the results from the two treatment centres considered. This might have been due the scarcity of observations towards the end of trials, the immune system of children may also contribute prolong time to first recurrence, the amount of mosquitoes and exposure of children. Despite this shortcoming, the artemisinin-based combination therapy performed well in both Konde and Uzini. For the 42 days period, ASP had a $55 \%$ probability of successfully treating patients and $71 \%$ success probability, if followed for 84 days. Similarly in Uzini, the probability was $94 \%$ for a 42 days treatment period and $87 \%$, if patients were treated for 84 days.

Our methodology produced estimates and posterior distributions for the duration in days ASP could postpone a recurrence of malaria. In case of treatment failure recipients of ASP, will stay asymptomatic for 6.2 days if treatment was followed for 42 days. Meanwhile, the malaria free duration will be 5.4 days if treatment was provided for 84 days. This is a benefit and a major contribution to existing knowledge on the efficacy of malaria treatment studies.

We assumed uniform distributions for everyone within intervals before follow-up dates, which is a limitation. However, in practice we can only know an outcome on the screening date and nothing in between. A more comprehensive and generalized methodology is possible with the inclusion of other background variables such as age and weight of patients.

\section{ACKNOWLEDGEMENT}

The author is thankful to the International Science Programme (ISP), Uppsala, Sweden for the financial support. I am equally grateful to the Department of Infectious Diseases of the Karolinska Institute, Sweden for providing the data.

\section{REFERENCES}

[1] Spiegelhalter DJ, Freedman LS, Parmar MKB. Bayesian approaches to randomized trials. J Roy Statist Soc Ser A 1994; 357-16. http://dx.doi.org/10.2307/2983527

[2] Fisher LD. Comments on: Bayesian and frequentist analysis and interpretation of clinical trials. Control Clin Trials 1996; 17(5): 423-34.

http://dx.doi.org/10.1016/S0197-2456(96)00043-8 
[3] Freedman L. Bayesian statistical methods. BMJ 1996; 313(7057): 569-70. http://dx.doi.org/10.1136/bmj.313.7057.569

[4] Woodworth GG. Biostatistics: A Bayesian Introduction. WileyInterscience 2004; vol. 499.

[5] von Seidlein L, Milligan P, Pinder M, Bojang K, Anyalebechi $\mathrm{C}$, Gosling R, et al. Efficacy of artesunate plus pyrimethamine-sulphadoxine for uncomplicated malaria in Gambian children: a double-blind, randomised, controlled trial. Lancet 2000; 355(9201): 352-57. http://dx.doi.org/10.1016/S0140-6736(99)10237-X

[6] Zwang J, Olliaro $P$, Barennes $H$, Bonnet $M$, Brasseur $P$, Bukirwa $\mathrm{H}$, et al. Efficacy of artesunate-amodiaquine for treating uncomplicated falciparum malaria in sub-Saharan Africa: a multi-centre analysis. Malar J 2009; 8(1): 203. http://dx.doi.org/10.1186/1475-2875-8-203

[7] Mutabingwa TK. Artemisinin-based combination therapies (ACTs): Best hope for malaria treatment but inaccessible to the needy. Acta Trop 2005; 95(3): 305-15. http://dx.doi.org/10.1016/j.actatropica.2005.06.009

[8] Mårtensson A, Strömberg J, Sisowath C, Msellem MI, Gil JP, Montgomery SM, et al. Efficacy of artesunate plus amodiaquine versus that ofartemether-lumefantrine for the treatment of uncomplicated childhood Plasmodium falciparum malaria in Zanzibar, Tanzania. Clin Infect Dis 2005; 41(8): 1079-86. http://dx.doi.org/10.1086/444460

[9] Mårtensson A, Ngasala B, Ursing J, Veiga MI, Wiklund L, Membi C, et al. Influence of consecutive-day blood sampling on polymerase chain reaction-adjusted parasitological cure rates in an antimalarial-drug trial conducted in Tanzania. $J$ Infect Dis 2007; 195(4): 597-601. http://dx.doi.org/10.1086/510910

[10] Kum CK. Bayesian analysis of two malaria treatments and probability modeling of malaria parasite genotypes, (PhLic Thesis). Stockholm University 2009.

[11] Greenhouse B, Dokomajilar C, Hubbard A, Rosenthal PJ, Dorsey G. Impactof transmission intensity on the accuracy of genotyping to distinguish recrudescence from new infection in antimalarial clinical trials. AAC 2007; 51(9): 3096-103.

[12] Sowunmi A, Adewoye EO, Gbotosho GO, Happi CT, Sijuade $A$, Folarin $\mathrm{OA}$, et al. Factors contributing to delay in parasite clearance in uncomplicated falciparum malaria in children. Malar J 2010; 9: 53.

http://dx.doi.org/10.1186/1475-2875-9-53

[13] White NJ. The parasite clearance curve. Malar J 2011; 10(1): 278.

http://dx.doi.org/10.1186/1475-2875-10-278

[14] Datta S. Estimating the mean life time using right censored data. Statist Methodol 2005; 2(1): 65-69.

http://dx.doi.org/10.1016/j.stamet.2004.11.003

[15] Zhong $M$, Hess KR. Mean Survival Time from Right Censored Data. Collection of Biostatistics Research Archives-bepress 2009.

[16] Ying G, Heitjan DF, Chen TT. Nonparametric prediction of event times in randomized Clinical trials. 2004; 1(4): 352-61. http://dx.doi.org/10.1191/1740774504cn030oa

[17] Demiris N, Lunn D, Sharples LD. Survival extrapolation using the poly-Weibull model. Stat Methods Med Res 2011; 0(0): 115.

http://dx.doi.org/10.1177/0962280211419645

[18] Gbotosho GO, Okuboyejo TM, Happi CT, Sowunmi A. Recrudescent Plasmodium falciparum infections in children in an endemic area following artemisinin-based combination treatments: Implications for disease control. Asian Pac J Trop Dis $2011 ; 1(3)$ : 195-202. http://dx.doi.org/10.1016/S2222-1808(11)60027-3

[19] Box GEP, Tiao GC. Bayesian inference in statistical analysis. DTIC Document 1973.

[20] Bernardo JM, Smith AFM. Bayesian theory. Meas Sci Technol 2001; 12(2): 221. http://dx.doi.org/10.1088/0957-0233/12/2/702

[21] Gelman A, Carlin JB, Stern HS, Rubin DB. Bayesian data analysis. Chapman \& Hall/CRC 2003.

[22] Spiegelhalter DJ, Abrams KR, Myles JP. Bayesian approaches to clinical trials and health-care evaluation. Wiley 2004; vol. 13

[23] Lesaffre E, Lawson AB. Introduction to Bayesian Inference. Bayesian Biostatistics 2012; pp. 122-124.

[24] Chin R, Lee BY. Principles and practice of clinical trial medicine.Academic Press 2008.

Received on 05-02-2013

Accepted on 10-04-2013

Published on 30-04-2013

http://dx.doi.org/10.6000/1929-6029.2013.02.02.01

(c) 2013 Kum et al.; Licensee Lifescience Global.

This is an open access article licensed under the terms of the Creative Commons Attribution Non-Commercial License (http://creativecommons.org/licenses/by-nc/3.0/) which permits unrestricted, non-commercial use, distribution and reproduction in any medium, provided the work is properly cited. 